\title{
ARTICLE
}

\section{Nonlinear photocarrier dynamics and the role of shallow traps in mixed-halide mixed-cation hybrid perovskites ${ }^{\dagger}$}

Received 00th January 20xx, Accepted 00th January 20xx DOI: $10.1039 / x 0 \times x 00000 x$

David A. Valverde-Chávez, ${ }^{\mathrm{a}} \S$ Esteban Rojas-Gatjens, ${ }^{\mathrm{a}} \S$ Jacob Williamson, ${ }^{\mathrm{b}}$ Sarthak Jariwala, ${ }^{\mathrm{cd}}$

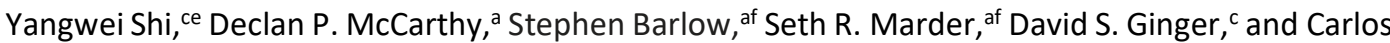
Silva-Acuña*abf

\begin{abstract}
We examine the role of surface passivation on carrier trapping and nonlinear recombination dynamics in hybrid metal-halide perovskites by means of excitation correlation photoluminescence (ECPL) spectroscopy. We find that carrier trapping occurs on subnanosecond timescales in both control (unpassivated) and passivated samples, which is consistent within a shallowtrap model. However, the impact of passivation has a direct effect on both shallow and deep traps. Our results reveal that the effect of passivation of deep traps is responsible for the increase of the carrier lifetimes, while the passivation of shallow traps reduces the excitation density required for shallow-trap saturation. Our work demonstrates how ECPL provides details about the passivation of shallow traps beyond those available via conventional time-resolved photoluminescence techniques.
\end{abstract}

\section{Introduction}

Over the last decade, hybrid organic-inorganic perovskites (HOIPs) have emerged as promising low-defect-density semiconductors with useful applications in optoelectronics, such as solar cells ${ }^{1-4}$, light-emitting diodes ${ }^{5,6}$, and lasers ${ }^{7,8}$. In particular, solar cell devices have reached remarkable power conversion efficiencies (PCEs); many NREL-validated PCEs for single-junction cells are above $20 \%$ with the highest PCE over 25\%. ${ }^{9}$ However, non-radiative charge-carrier recombination prevents HOIPs from approaching their theoretical efficiency. It has been shown that single-crystalline lead-halide materials possess a high tolerance to intrinsic defects ${ }^{10}, 11$. The interfaces and grain boundaries of perovskite films have been identified as the source of the dominant trap-assisted nonradiative recombination ${ }^{12-14}$. Different post-processing passivation strategies have proven effective in reducing the nonradiative pathways, namely passivation by oxygen exposure ${ }^{15}$, peroxide treatment ${ }^{16}$, and surface modifiers ${ }^{12}, 17$. Characterization of the effect of the passivation molecule on the surface is usually limited to time-resolved photoluminescence measurements and PCE comparisons between devices, which affords limited

\footnotetext{
a. School of Chemistry and Biochemistry, Georgia Institute of Technology, Atlanta, GA 30332, USA. E-mail: carlos.silva@gatech.edu.

b. School of Physics, Georgia Institute of Technology, Atlanta, GA 30332, USA

c. Department of Chemistry, University of Washington, Seattle, WA 98195, USA

d. Department of Materials Science and Engineering, University of Washington, Seattle, WA 98195, USA.

e. Molecular Engineering \& Sciences Institute, University of Washington, Seattle, WA 98195, USA.

f. School of Materials Science and Engineering, Georgia Institute of Technology, Atlanta, GA 30332, USA.

$\S$ DAVC and ERG are first co-authors of this manuscript.

tElectronic Supplementary Information (ESI) available: See DOI: 10.1039/ $\mathrm{x} 0 \mathrm{xx} 00000 \mathrm{x}$
}

insight into the photophysical nature of carrier trapping processes in HOIPs.

Several physical models have been proposed previously to describe trap-assisted nonradiative recombination for leadhalide perovskites following the Shockley-Read-Hall (SRH) formalism. ${ }^{18,19}$ Stranks et al. describe the interplay between excitons and subgap states in $\mathrm{CH}_{3} \mathrm{NH}_{3} \mathrm{Pbl}_{3-\mathrm{x}} \mathrm{Cl}_{x}$; their model proposed the presence of deep traps by means of background doping caused by the photoinduced filling of traps which exhibit nonradiative recombination with very slow dynamics ${ }^{20}$. Dobrolovsky et al. concluded a very different scenario for the same material in which there are distinct types of traps, some of which are "shallow" and emissive ${ }^{21}$. Several authors have noted the importance of considering shallow traps as part of the photophysical description of perovskite nanocrystals, suggesting that trapping and detrapping mechanisms are responsible for the long-lived photoluminescence traces ${ }^{22,23}$. Although, it is not believed that shallow traps are responsible for the long-lived photoluminescent trace in thin films ${ }^{24}$. Recently, shallow traps have been reported to be responsible for degradation of $\mathrm{FAPbl}_{3}{ }^{25}$.

Because the processing conditions and identities of precursors have been shown to affect the defect physics ${ }^{21,26}$, not only by changing the trap density, but also the intrinsic characteristics of the trap (e.g., trap depth, trapping rate). Here we implement a time-resolved spectroscopic method that characterises carrier trapping dynamics beyond common techniques such as time- 
(a)

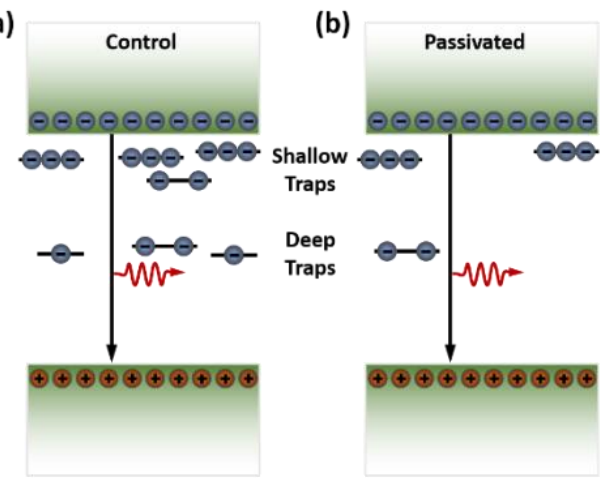

(c)

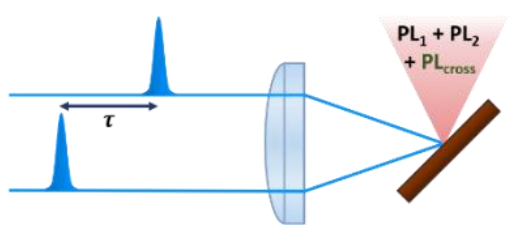

Fig. 1: Conceptual. Schematic representation of shallow trap model with (a) high trap density in a control sample and (b) low trap density in a passivated sample. (c) Schematic of the ECPL pulse sequence and PL terms.

resolved photoluminescence spectroscopy. Specifically, we employ excitation correlation photoluminescence (ECPL) spectroscopy, which was originally used to describe population mixing dynamics of GaAs p-type semiconductors ${ }^{27,28}$ as well as picosecond carrier lifetimes in other inorganic semiconductors ${ }^{29-33}$, and exciton dynamics in carbon nanotubes ${ }^{34,35}$. More recently, it was used to characterise the defect physics in $\mathrm{CH}_{3} \mathrm{NH}_{3} \mathrm{PbBr}_{3}$ thin films and $\mathrm{CsPbBr}_{3}$ nanocrystals ${ }^{26}$. The technique consists of exciting the sample with two ultrafast pulses modulated at different frequencies $\Omega_{1}, \Omega_{2}$. The measured photoluminescence intensity then includes contributions from recombination between populations generated by the first pulse, the second pulse, and a mixed population term; this mechanism is represented schematically in Fig. 1c. The contribution of the mixed population to the measured photoluminescence intensity is described by:

$$
P L_{\text {cross }}=\int_{\tau}^{\infty}[n(t) p(t-\tau)+n(t-\tau) p(t)] d t .
$$

By using lock-in heterodyne detection, the signal is demodulated at the sum of the pulse modulation frequencies $\left|\Omega_{2}+\Omega_{1}\right|$ to isolate this contribution. ECPL is well-suited for measuring sub-nanosecond short-carrier lifetimes without employing any complex electronics for ultrafast detection. Specifically, it is useful in characterizing nonradiative pathways, as was shown by Johnson et al. ${ }^{29}$. Recombination dynamics in which the radiative pathways are predominant results in an ECPL signal of zero. Meanwhile, the signal for ECPL for a trapping-dominated process can be as high as $30 \%$ of the total signal ${ }^{29}$
In this work, we study the effect of passivation with APTMS, 3-aminopropyl)trimethoxysilane, on the defect scenario of control perovskite thin films with composition $\mathrm{FA}_{0.83} \mathrm{Cs}_{0.17} \mathrm{~Pb}\left(\mathrm{I}_{0.85} \mathrm{Br}_{0.15}\right)_{3}$, employing both ECPL spectroscopy and time-resolved photoluminescence measurements. We describe the defect scenario as a mixture of deep traps and shallow traps. We observe fast ECPL photocarrier dynamics at room temperature for both the passivated and the control sample, suggesting a fast carrier trapping rate resulting from shallow traps. The effect of passivation is observed as a decrease in trap density of both deep traps and shallow traps. This is also supported by temperature-dependent ECPL experiments, which show a shift to a lower excitation threshold in trap saturation. By numerically solving the differential rate equations for both shallow and deep trap cases, we give a qualitative description of the features observed at low temperature, here assigned to Auger recombination.

\section{Experimental section}

\section{$\mathrm{FA}_{0.83} \mathrm{Cs}_{0.17} \mathrm{~Pb}\left(\mathrm{I}_{0.85} \mathrm{Br}_{0.15}\right)_{3}$ thin film preparation and passivation.}

Film preparation and passivation was performed according to the procedure described by Jariwala et al. ${ }^{36}$ All precursors were purchased from Sigma, unless stated otherwise. Perovskite precursor solutions $(1 \mathrm{M})$ were formed by adding stoichiometric amounts of precursor salts formamidinium iodide (greatcellsolar), $\mathrm{Csl}, \mathrm{Pbl}_{2}$ (Sigma), and $\mathrm{PbBr}_{2}$ in $\mathrm{DMF}: \mathrm{DMSO}$ (1300:640). $60 \mu \mathrm{L}$ of formamide was added to make a $2 \mathrm{~mL} 1 \mathrm{M}$ solution (with $3 \mathrm{v} \%$ formamide). The substrates were plasma cleaned before spin coating and the perovskite solution was filtered before deposition (inside the glovebox). $\sim 25 \mu \mathrm{L}$ of the filtered perovskite solution was deposited on top of the substrate. The substrates were spun at $4000 \mathrm{rpm}$ for 60 seconds. When $\sim 35 \mathrm{~s}$ was remaining, $\sim 50 \mu \mathrm{L}$ of chlorobenzene (CB) was dropped from above. The films were then annealed on the hotplate at $100{ }^{\circ} \mathrm{C}$ for $30 \mathrm{~s}$ and at $150{ }^{\circ} \mathrm{C}$ for $10 \mathrm{~min}$. Surface passivation with APTMS was done in a vacuum oven (Precision Vacuum Oven Model 19) for 5-10 min at room temperature under vacuum with the gauge pressure reading -30 " $\mathrm{Hg}$ relative to atmospheric pressure. For further details refer to ref. ${ }^{36}$ The films of untreated (herein called $\mathrm{Cs} 17 \mathrm{Br} 15$ control, or just control) and APTMS-treated $\mathrm{FA}_{0.83} \mathrm{Cs}_{0.17} \mathrm{~Pb}\left(\mathrm{I}_{0.85} \mathrm{Br}_{0.15}\right)_{3}$ were prepared at the University of Washington and shipped to Georgia Tech for ECPL characterization. A detailed characterisation of the bandgap, X-ray diffraction, and composition can be found in ref $^{36}$. It is worth mentioning that the characterisation of the surface composition, by XPS and band edge, was cross-validated and showed to be reproducible after the shipping at multiple institutions. ${ }^{36}$

\section{UV-Vis Absorption and steady-state Photoluminescence.}

UV-Vis absorption spectra were measured using an Agilent 8453 UV-Vis spectroscopy system, the data corrected for scatter at the longest wavelengths. The PL spectrum was also acquired in the same configuration as the ECPL instrument setup (described 
below) by collecting the emission with an optical fiber coupled to a spectrometer (USB2000+, Ocean Optics).

\section{Time-Resolved Photoluminescence (TRPL).}

TRPL was measured using a Picoharp 300 TCSPC system equipped with a $470 \mathrm{~nm}$ pulsed diode laser (PicoQuant PDL-800 LDH-P-C-470B, 300 ps pulse width). The laser was pulsed at repetition rates from $250 \mathrm{KHz}$ to $1 \mathrm{MHz}$. The $\mathrm{PL}$ emission was filtered using a $580 \mathrm{~nm}$ long-pass filter before being directed to the detector.

\section{Excitation Correlation Photoluminescence Spectroscopy (ECPL).}

A schematic representation of the ECPL setup is shown in Fig. S1. In the ECPL instrument setup, $1030 \mathrm{~nm}, \sim 220 \mathrm{fs}$ pulses are generated in an ultrafast laser system at a $100 \mathrm{kHz}$ repetition rate (PHAROS Model PH1-20-0200-02-10, Light Conversion). A portion of the laser beam is sent into a commercial optical parametric amplifier (ORPHEUS, Light Conversion), where a 2.64-eV pump excitation pulse is selected. Typical excitation densities used in the experiment range from $3 \times 10^{16}$ to $6 \times 10^{18}$ $\mathrm{cm}^{-3}$. The excitation intensity is varied by moving a motorized filter wheel (FW212CNEB, Thorlabs). The pulse trains are then split $50 / 50$ by a beam splitter cube, where one of the beams is directed to a motorized linear stage (LTS300, Thorlabs), allowing for control of the delay between the two pulses. Each pulse is modulated with a chopper at the frequencies of 373 and $199 \mathrm{~Hz}$, respectively, and the pulses are then focused onto the perovskite sample with a $150-\mathrm{mm}$ focal length lens. The emitted $\mathrm{PL}$ is filtered with a 550-nm long-pass filter to get rid of the pump, then it is focused into a photoreceiver. The photoreceiver is connected to a lock-in amplifier (HF2LI, Zurich Instruments) where the total integrated $\mathrm{PL}$ and the nonlinear component $\triangle \mathrm{PL}$ are obtained simultaneously by demodulating both at the fundamental and the sum frequency. The perovskite films are measured inside a closed-cycle cryostat (Montana Instruments). It is worth mentioning that some degradation was observed at the highest fluences. For all measurements we acquired data from the lowest to highest excitation fluence, all in the same spot.

\section{Results and Discussion}

\section{Linear Spectroscopy}

The PL and absorption spectra for the control and passivated samples are shown in Fig. 2a. From UV-vis absorption spectroscopy, we determine the band gap to be $1.63 \mathrm{eV}$ from Tauc plots. From PL spectra, the control samples exhibit a maximum emission energy peaked at $1.63 \mathrm{eV}$ on average, while the passivated samples exhibit a maximum emission energy $1.60 \mathrm{eV}$ on average. Recently, different lineshapes of PL spectra have been explained in the context of PL reabsorption ${ }^{37,} 38$, specifically an apparent redshift caused by the reabsorption and the PL propagation along the film. Under this interpretation the $\mathrm{PL}$ in the passivated sample has higher photo recycling, expected from a sample low trap density. (a)

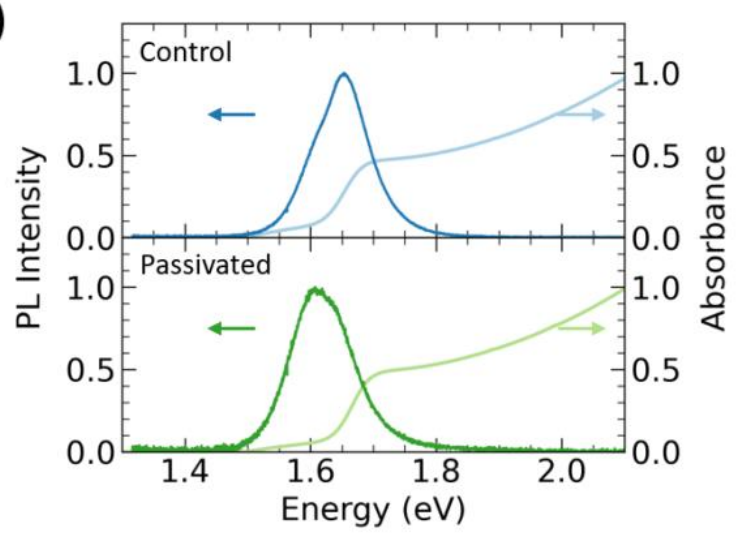

(b)

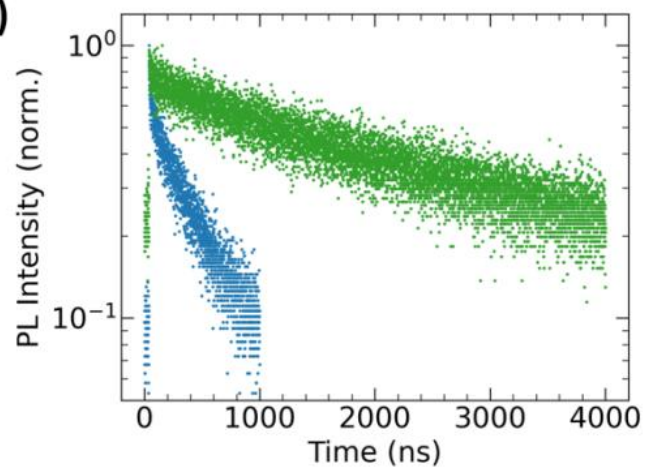

Fig. 2: Absorbance, PL and TRPL. (a) Absorbance and photoluminescence of a control and passivated samples. (b) Time resolved PL for control (blue) and ATPMS passivated (green) sample.

The APTMS vapour passivation procedure has been demonstrated to reduce non-radiative recombination, increase average PL lifetimes by over an order of magnitude, and increase the external quantum yield by a factor of $60^{36}$. Due to the effect of APTMS on surface defects, this system is ideal to describe the effect of passivation on the nature of traps and nonradiative recombination dynamics. Typical time-resolved PL measurements for control and passivated samples, collected at a low fluence of $<50 \mathrm{~nJ} / \mathrm{cm}^{2}$ per pulse, are shown in Fig. $\mathbf{2 b}$. The $\mathrm{PL}$ average lifetime $\langle\tau\rangle$ increases after APTMS deposition in agreement with previous reports, ${ }^{36}$ and is attributed to the surface passivation of traps. Along with an increase in the PL average lifetime, we also observe an increase in the $\beta$ parameter when fitting TRPL to a stretched exponential function, defined by equations 2 and 3 . The increase in the $\beta$ parameter suggests a more homogenous distribution of kinetics after APTMS passivation ${ }^{39}$, which we interpret as a distribution of trap environments, consistent with reports by Jariwala et. $a^{\beta 6}$.

$$
\begin{gathered}
I=I_{0} e^{-\left(\frac{t}{\tau_{c}}\right)^{\beta},} \\
\langle\tau\rangle=\frac{\tau_{c}}{\beta} \Gamma\left(\frac{1}{\beta}\right) .
\end{gathered}
$$



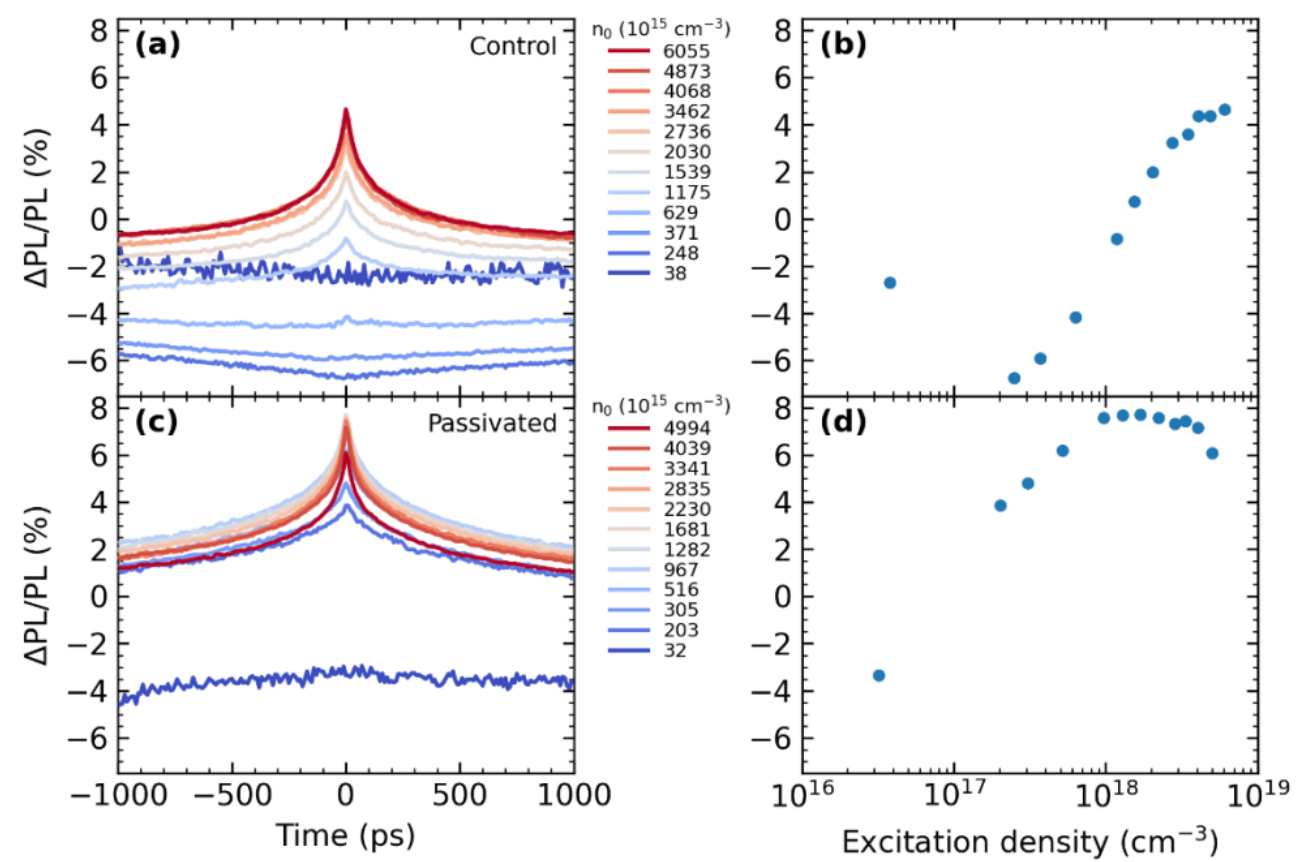

Fig. 3: ECPL dynamics at 295K ECPL dynamics at $295 \mathrm{~K}$ of (a) Cs17Br15 control and (c) APTMS passivated perovskite. ECPL at $t=0 \mathrm{ps}$ for (b) Cs17Br15 control and (d) APTMS passivated perovskites.

\section{Room temperature ECPL}

Fig. 3 shows fluence-dependent ECPL acquired at room temperature for both $\mathrm{FA}_{0.83} \mathrm{Cs}_{0.17} \mathrm{~Pb}\left(\mathrm{I}_{0.85} \mathrm{Br}_{0.15}\right)_{3}$ control and APTMS passivated films. We start by discussing the ECPL dynamics on the control sample Fig. 3a. At the lowest excitation density, the ECPL signal is negative and flat; for the next excitation density it becomes more negative and then monotonically increases with excitation intensity. At a higher excitation density $\left(n_{D} \sim 1 \times 10^{18} \mathrm{~cm}^{-3}\right)$ the ECPL signal develops dynamics indicative of a picosecond process due to fast charge trapping ${ }^{31}$. At the highest excitation densities, the system reaches a saturation point. This behaviour can be explained by the presence of a high trap density that is only filled at a high excitation density $\left(\mathrm{n}_{\mathrm{D}} \sim 5 \times 10^{18} \mathrm{~cm}^{-3}\right)$. For the APTMS passivated sample, the ECPL photocarrier dynamics in Fig. 3c share some similarities with the control sample, but the saturation threshold changes due a lower trap density. The ECPL also starts flat and negative, but the next fluence already has dynamical features and the overall signal saturates at lower fluences. This is expected, given that the passivation procedure would decrease the density of traps causing saturation to occur at lower excitation densities. In this case, we observe a small decrease of the signal at high excitation densities. We attribute this to Auger recombination starting to play a role in the dynamics as we approach the saturation threshold but not enough to observe a significant decrease.

It is useful to look at the ECPL ( $t=0)$ signal, which corresponds to the situation when the two excitation pulses arrive at the same time, to visualise the effect that traps have on the ECPL saturation. Fig. 3b shows ECPL $(t=0)$ signal for the control sample, which increases until it saturates at $\mathrm{n}_{\mathrm{D}} \sim 5 \times 10^{18} \mathrm{~cm}^{-3}$. In Fig. 3d, the ECPL $(t=0)$ signal from the passivated sample initially is negative and then grows until saturation at around $\mathrm{n}_{\mathrm{D}} \sim 1 \times 10^{18} \mathrm{~cm}^{-3}$, and finally decreases due to Auger recombination. These ECPL photocarrier dynamics on control and passivated samples are consistent with a shallow trap model reported before on $\mathrm{CH}_{3} \mathrm{NH}_{3} \mathrm{Pbl}_{3}$ perovskites ${ }^{26}$ since they present a picosecond charge trapping process. If the fast trapping were caused by deep defects, then we would expect a sub-nanosecond short recombination lifetime which is inconsistent with the photoluminescence trace observed experimentally, Fig. S11 and S12 show that the population evolution during first nanosecond for deep traps is negligible. Note that this does not imply that deep traps are not present, simply that they are not responsible for the observed positive dynamics. We note that the excitation density values at which the ECPL saturation is observed are slightly higher than previous trap density values reported for similar perovskites ${ }^{20}, 39$. However, it is known that the saturation threshold for thermally activated traps is dependent on the trapping/detrapping equilibrium, as will be discussed below.

\section{Temperature-Dependent ECPL experiments}

There are several reports in the literature on the effect of temperature on recombination photophysics in lead-halide perovskites. Stranks et al. ${ }^{20}$ reported a change in the trap density with temperature and speculated the origin was due to thermally activated traps for $\mathrm{MAPbl}_{3}$ films. Kandada et al. ${ }^{26}$ also reported thermally activated doping in $\mathrm{MAPbBr}_{3}$. It is also 


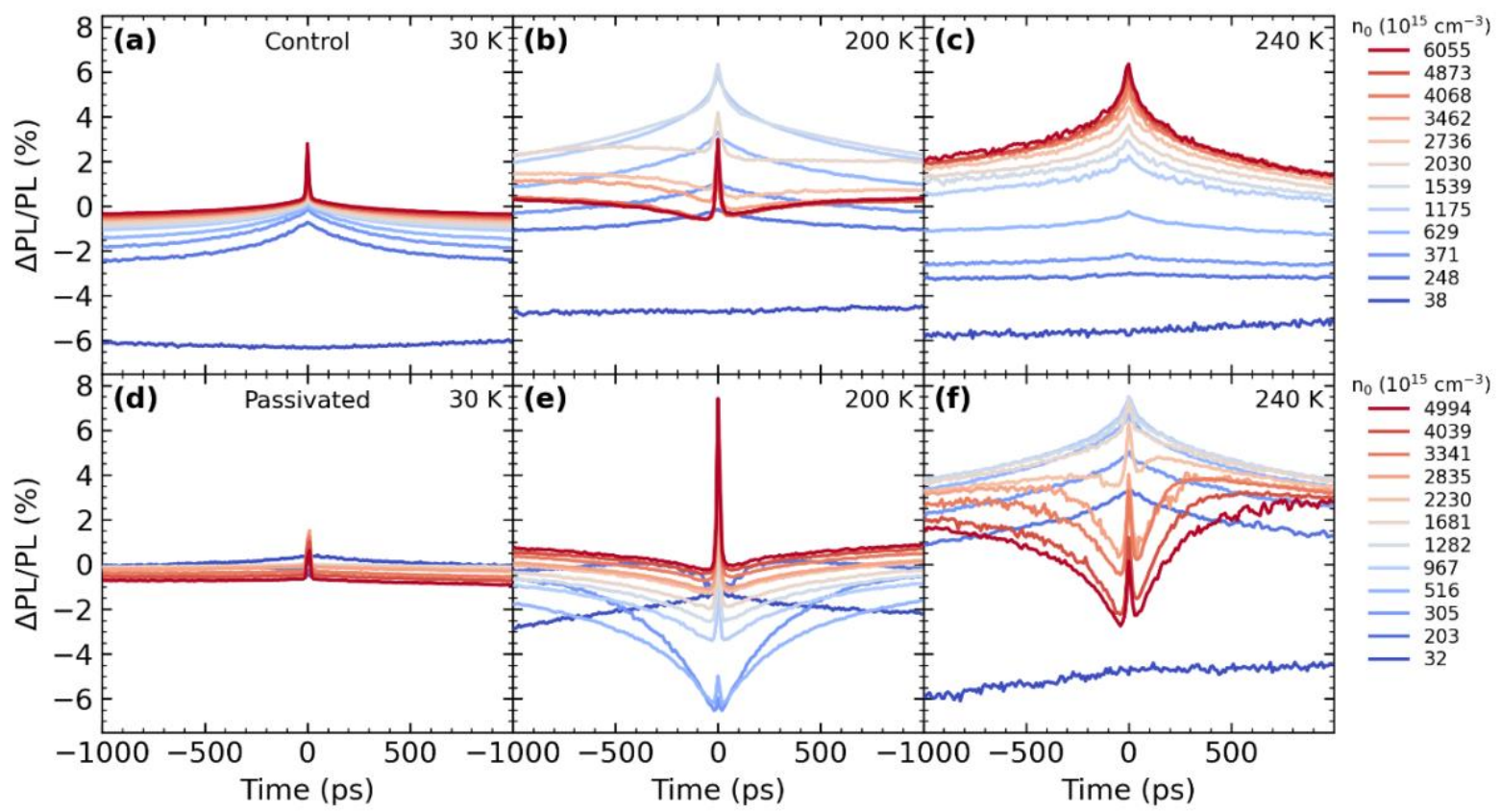

Fig. 4: ECPL Temperature dependence. ECPL dynamics for Cs17Br15 control at temperature of (a) $30 \mathrm{~K}$ (b) $200 \mathrm{~K}$ and (c) $240 \mathrm{~K}$. ECPL dynamics for an APTMS passivated perovskite at (d) $30 \mathrm{~K}$ (e) $200 \mathrm{~K}$ and (f) $240 \mathrm{~K}$.

worth noting that the detrapping rate is temperature dependent. Therefore, understanding the effect of temperature on ECPL signal would shed light on the physics behind recombination processes. For this reason, we measured ECPL at various temperatures (Fig. 4, S4 and S5).

We start by discussing the temperature dependence of ECPL photocarrier dynamics for the control sample. At $30 \mathrm{~K}$ (Fig. 4a), the ECPL starts negative and flat similar to the $295 \mathrm{~K}$ signal. However, dynamics soon develop as excitation density increases, with the ECPL signal approaching zero and appearing to saturate at the highest excitation density. Furthermore, close to time zero with excitation density $n_{D}>1 \times 10^{18} \mathrm{~cm}^{-3}$, ultrafast dynamics appear. In the context of the shallow trap model proposed above, a decrease of the ECPL signal is expected since the trapped electron will lack sufficient thermal energy to hop from the trap state to the conduction band, which will decrease the fluence threshold at which saturation occurs and allow Auger recombination to occur. ECPL photocarrier dynamics at $200 \mathrm{~K}$ are complex for the control sample (Fig. 4b) pointing to multiple overlapping regimes. The signal again starts negative and flat at the lowest fluence, then increases with dynamics similar to the $295 \mathrm{~K}$. However, at an excitation density $\mathrm{n}_{\mathrm{D}} \sim 2 \times 10^{18} \mathrm{~cm}^{-3}$, the dynamics turn negative due to Auger recombination and, again, ultrafast dynamics appear. Further increasing the temperature to $240 \mathrm{~K}$ (Fig. 4c) leads to ECPL dynamics that are similar to those at $295 \mathrm{~K}$.

We now discuss the ECPL signal for the passivated sample. At 30 $\mathrm{K}$ (Fig. 4d), the ECPL signal is close to zero even at the lowest fluences. As the excitation density increases, the ECPL signal decreases, though to a minuscule extent. In this case, if Auger recombination becomes predominant as is expected for very low temperatures, it is also expected that the ECPL intensity will continue to decrease to negative values as the excitation density continues to increase. It is important to note that the picosecond dynamics appear at a much lower threshold. Increasing the temperature to $200 \mathrm{~K}$ (Fig. 4e) shows predominantly negative signal at the lowest fluences, with ultrafast dynamics becoming more prominent along with an overall increase in ECPL signal as excitation density increases. Furthermore, an increase to $240 \mathrm{~K}$ (Fig. 4c) leads to dynamics like the ones at $295 \mathrm{~K}$ for lower excitation density. That is, the signal starts negative and flat at the lowest fluence, then becomes positive until $\mathrm{n}_{\mathrm{D}} \sim 2 \times 10^{18} \mathrm{~cm}^{-3}$, after which the dynamics turn negative and the ultrafast dynamics peak appears. This is somewhat similar to the control at $200 \mathrm{~K}$ and further suggests that the appearance of this feature is caused by saturation. The temperature threshold then differs from control to passivated since the density of traps is different. The negative curvature dynamics in the ECPL signal have been reported for hexagonal $\mathrm{GaN}^{32}$ and was attributed to population saturation.

A narrow peak appears in most of the ECPL photocarrier dynamics as the excitation density is increased, as we can see from Fig. 4. We believe this is due to an effect termed amplification of spontaneous emission (ASE) ${ }^{40}$. It is optical gain due to light trapped in a waveguide, and air-perovskite-glass interfaces may act as such a waveguide. Interestingly, the presence of this feature is temperature-dependent and at the same time correlates with the very fast dynamics and negative dynamics observed in the ECPL. The correlation also holds when we measure the passivated sample: ASE remains present at higher temperatures (Fig. S2 and S3), and the excitation densities needed to show ASE are much lower. 
When we look at the ECPL $(t=0)$ for the temperature dependence (Fig. S6 and S8), we find that there are two dominant regimes. For temperatures $240 \mathrm{~K}$ and above, the ECPL signal increases monotonically with fluence and reaches a saturation point, the saturation point is lower for the passivated sample hinting a lower shallow trap energy, as discussed above. But besides, the saturation threshold seems to shift to lower excitation as the temperature is decreased, this further supports the lower energy of the shallow trap. At $\mathrm{T}=200 \mathrm{~K}$ and below, there seems to be local maximum in the control sample at lower excitation intensities, this local maximum is closely related to the appearance of the ASE. This effect does not seem to be present for the passivated sample although it may very well be that we are just not covering the region as it will shift to lower excitation densities. It is important to note that these features are present, albeit at a lesser degree when looking at a time away from zero to avoid the ASE peak, (ECPL ( $t=250 \mathrm{ps})$ in Fig. S7 and S9).

\section{Photophysical model and simulation description}

The ECPL results obtained are interpreted following the model for shallow traps described by the rate equations shown below:

$$
\begin{aligned}
& \frac{d n}{d t}=P-B n p-\gamma_{t} n\left(N_{t}-n_{t}\right)+\gamma_{d} n_{t}-\gamma_{\text {Auger }} n^{2} p, \\
& \frac{d p}{d t}=P-B n p-\gamma_{n r} n_{t} p-\gamma_{\text {Auger }} n^{2} p, \\
& \frac{d n_{t}}{d t}=\gamma_{t} n\left(N_{t}-n_{t}\right)-\gamma_{d} n_{t}-\gamma_{n r} n_{t} p,
\end{aligned}
$$

Where $\gamma_{t}$ is the trapping rate, $B$ is the bimolecular recombination rate, $N_{t}$ is the density of traps, $\gamma_{d}$ is the detrapping rate, $\gamma_{n r}$ is the nonradiative recombination rate, and $\gamma_{\text {Auger }}$ is the Auger recombination rate. $P$ corresponds to the photocarriers generated by the pulses. The pulses are considered to interact instantaneously both for the interpretation of the results and for the simulation results. The detrapping rate $\left(\gamma_{d}\right)$ is of particular interest as it relates to the depth of traps. It is described as ${ }^{18,26 \text { : }}$

$$
\gamma_{d}=\gamma_{t} \cdot 2\left(\frac{m_{e}^{*} k_{b} T}{2 \pi h^{2}}\right)^{\frac{3}{2}} e^{-\frac{\Delta E_{b}}{k_{b} T}}
$$

The temperature dependence study of recombination parameters has been reported for $\mathrm{CH}_{3} \mathrm{NH}_{3} \mathrm{Pbl}_{3}{ }^{41}$ by using the model $\dot{P} L=-k_{1} n-k_{2} n^{2}-k_{3} n^{3}$. This model does not capture the diversity of processes occurring nor their reversibility. The bimolecular recombination rate was shown to change with temperature by two orders of magnitude between $160 \mathrm{~K}$ and $300 \mathrm{~K}$. The Auger rate remained constant, with a significant increase at temperatures $<150 \mathrm{~K}$ related to a change in structure. At temperatures lower than $160 \mathrm{~K}$, a change in the photoluminescence lineshape was observed, indicative of a phase transition from tetragonal to orthorhombic structure. We do not observe such signatures (Fig. S2 and S3) in concordance

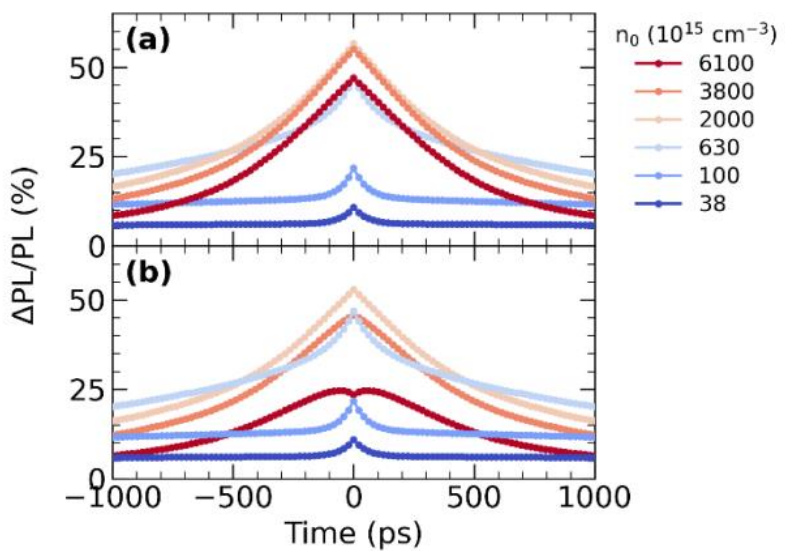

Fig 5. Simulation of ECPL shallow traps with an Auger recombination rate of (a) $10^{-28} \mathrm{~cm}^{6} / \mathrm{s}$ and (b) $10^{-27} \mathrm{~cm}^{6} / \mathrm{s}$.

with phase transition quenching observed for $(\mathrm{Cs}, \mathrm{MA}, \mathrm{FA}) \mathrm{Pb}(\mathrm{Br}, \mathrm{I})_{3}{ }^{42}$. Due to this observation, we assume that all our measurements were taken for the same phase, therefore we do not consider structural effects as the source of changes in the ECPL experiments. In the temperature-dependent simulation, we assume the various parameters are constant and that the parameters in equation (7) are the only ones affecting the detrapping rate.

The binding energy for excitons has been estimated for leadhalide perovskites to be 6-60 meV43-48. Even at room temperature, the role of excitons cannot be overlooked, as they are constantly forming and dissociating ${ }^{20}$. However, we do not include exciton formation in the rate equations to avoid the over-parametrization of the model. This is justified as, in the context of ECPL experiments, the pairing and further recombination of generated photocarriers do not result in a change of the integrated photoluminescence measured by the photodetector, therefore the ECPL response will not be affected by this assumption and recombination from excitons can be safely ignored.

Our simulation of the ECPL signal solves the initial value problem created by the rate equations following a pulse interaction. This problem is then solved repeatedly over a range of delays and processed to extract simulated ECPL data. As the difference between positive and negative delay times is strictly due to the time-ordering of the pulses, the simulation only simulates positive delay times, and this result is then reflected across the zero-delay line to show negative delay times. This process is repeated for a defined set of parameters based on similar materials. The resulting images are either qualitatively compared to experimental data or studied to determine the qualitative effects of each parameter on ECPL data, depending on the exact simulation run and parameter sets chosen.

The simulation relies on several assumptions. Firstly, pulses are treated as though they interact instantaneously. This is a valid assumption to make as long as we recognise that the simulation will be inaccurate at timescales on the order of the pulse width. 
We also assume total absorption of the pulse to calculate the excitation density. This is inaccurate as we can observe remnants of the excitation pulse reflecting off the sample and transmitting through the sample. However, we are mainly interested in the qualitative behaviour at various excitation densities, so this is sufficient for our purposes.

\section{Qualitative description of the observed features.}

From the time-resolved photoluminescence data Fig. $\mathbf{2 b}$, the increase in photoluminescence lifetime suggests a decrease in the density of deeper traps between the control and passivated sample. We confirm the presence of shallow traps in both control and passivated films by ECPL at room temperature. The presence of distinct trap scenarios is expected, as thin film microstructure has proven to impact lifetimes of local carriers in the film domains $21,39,49,50$.

Next, we will justify the assignment of the various features, starting with a fast-trapping approximation. The shift of the saturation threshold with temperature is well-explained in the context of thermal detrapping. After the first pulse excitation, an ultrafast trapping and detrapping process will start. We assume that bimolecular and nonradiative recombination are significantly slower and, for sake of simplicity, a low enough excitation density such that the trapped electrons do not change $\left(N_{t}-n_{t}\right)<N_{t}$, these assumptions simplify the differential equation to an analytically solvable case. Then we can describe the ratio between free electron and trapped electron population evolution as:

$$
\frac{n}{n_{t}}=\frac{\gamma_{d}+N_{t} \gamma_{r} e^{-\left(\gamma_{d}+N_{t} \gamma_{r}\right) t}}{\left(\gamma_{r}-\gamma_{r} e^{-\left(\gamma_{d}+N_{t} \gamma_{r}\right) t}\right) N_{t}}
$$

For the sake of simplicity, we assume $t \rightarrow \infty$ :

$$
\frac{n}{n_{t}}=\frac{\gamma_{d}}{\gamma_{r} N_{t}}
$$

Recall that the temperature dependence is carried entirely by the $\gamma_{d}$ term. It is clear from equation (7) that the value of $\gamma_{d}$ decreases with temperature, resulting in a larger population of traps being filled. Even though the expression was obtained assuming low saturation, the trends discussed do not change.

The second pulse in the ECPL experiment acts as a probe of the evolution of the carrier populations caused by the first pulse. If the temperature is low, then most of the traps will be filled and the second pulse will result in high electron and hole densities, making Auger recombination and bimolecular recombination the dominant recombination pathways.

We can visualize the effect of the temperature in the saturation threshold by numerically solving the set of differential equations (2-4) and observing the carrier evolution during the first nanosecond, which is the range we are measuring in the ECPL experiment. This is equivalent to seeing the effect of just one pulse. From Fig S10, it can be observed that for the case of a shallow traps with a depth of $10 \mathrm{meV}$ as we decrease the temperature the trapped electron density increases faster, because of slower detrapping and this causes a decrease in the saturation threshold.

For a deeper shallow trap, $100 \mathrm{meV}$, the effect of temperature is almost negligible, Fig S10. This observation together with the difference of saturation threshold with temperature between control and passivated indicate that, from the initial distribution of shallow traps, the passivation procedure decreased the average trap depth.

We use the ECPL results obtained from the simulation to further support the assignment of the negative dynamics observed to the Auger recombination rate. Fig S12 shows the simulation of the ECPL response for the case of no traps and deep traps, for the case of no traps we observe an ECPL response of zero, meanwhile for deep traps the response without dynamics correlates with the previous experimental observation of deep traps in $\mathrm{MAPbBr}_{3}$ thin films ${ }^{26}$. In Fig 5, we show the result of the simulation for shallow traps with sub nanosecond trapping considering two different values of the Auger recombination rate. Note that at the highest fluence, negative dynamics are observed for the case with the higher $\gamma_{\text {Auger }}$. Furthermore, the and the trap dependent simulation (showed in Fig S13) support the argument of Auger recombination becoming predominant at high fluences. We recognize that the ECPL response obtained by the simulation is very high compared to the experiment. We attribute this difference to the inability of a rate equation model to include the statistical description of the defect scenario. Still, we consider the trends regarding fluence and temperature to provide significant information that aids and supports our interpretation of the ECPL response.

The ultrafast ECPL photocarrier dynamics near zero could also be in part due to hot carrier relaxation. Ultrafast hot carrier cooling has been reported in lead halide perovskites, however it is important to note that relaxation happens in ultrafast time scales less than $1 \mathrm{ps}^{51}$. The $\mathrm{ECPL}(\mathrm{t}=0)$ then might contain some contribution due to hot carriers, however the ultrafast transient has a FWHM of 20 ps. For this reason, another ultrafast process, like ASE must be involved. ASE would result in a positive feature in the ECPL response. This can be qualitatively rationalized by analysing the equation (10):

$$
P L_{\text {cross }}\left(I_{\text {pump }}, \tau\right)=P L\left(2 I_{\text {pump }}, \tau\right)-2 P L\left(I_{\text {pump }}, \tau\right)
$$

In this expression, the cross-correlation photoluminescence intensity is written as the difference between the PL generated by two pulses and twice the PL generated by one pulse. We expect the ASE to be higher for higher fluences therefore it will be more significant in the first term. ASE is a radiative pathway, then it becomes clear that cross-correlation PL is going to have a positive value. 


\section{Conclusions}

Herein, for $\mathrm{Cs}_{17} \mathrm{FA}_{83} \mathrm{~Pb}\left(\mathrm{I}_{85} \mathrm{Br}_{15}\right)$ passivated with APTMS, we distinguish between deep trap passivation and shallow trap passivation using excitation correlation photoluminescence spectroscopy. The sub-nanosecond recombination observed is evidence of the presence of shallow traps in both control and passivated samples. We interpret the change in the saturation threshold between the control and the passivated sample as a change of shallow trap density caused by the passivation procedure.

The correlation between the appearance of ASE in the temperature-dependent photoluminescence and shallow trap saturation suggests shallow traps play an important role by imposing a population inversion threshold. Targeted passivation of the shallow traps in high PLQY perovskite materials might help decrease the fluence threshold for lasing applications. This would be of particular interest in perovskite nanocavities with shallow traps, which present PLQY up to $90 \%{ }^{52}$. We also report the observation of Auger recombination dynamics observed when saturation is overcome. We speculate that the direct observation of Auger recombination will help study structure-property relations for lasing applications where Auger recombination dominates and limits the gain.

With a view to future work, since shallow trap passivation also plays a role in the electron mobility and their transport mechanism has been a recent subject of study ${ }^{53}$, this work also motivates the implementation of excitation correlation spectroscopy with photocurrent detection, which would expand the exploration of the passivation effect of electron/hole transport layers on the sub-nanosecond recombination in the functional devices.

\section{Author Contributions}

DAVC, ERG and JW constructed the ECPL setup. DAVC and ERG measured and analysed the ECPL data. JW, ERG and DAVC carried out simulations. SJ, YS and DPM processed the samples, supervised by DSG, SB and SRM. The intellectual basis for this paper was conceived by CS, DSG, SB and SRM. All co-authors participated in the redaction of the manuscript.

\section{Conflicts of interest}

The authors declare no conflicts of interest.

\section{Acknowledgements}

We acknowledge funding from the U.S. Department of Energy's Office of Energy Efficiency and Renewable Energy (EERE) under the Solar Energy Technologies Office Award Number DE-EE0008747. DPM acknowledges support from the Department of Education Graduate Assistance in Areas of National Need (GAANN) program at the Georgia Institute of Technology (Award \#P200A180075). CS

acknowledges equipment start-up funding from the College of Science of the Georgia Institute of Technology. The authors thank Prof. Ajay Ram Srimath Kandada for fruitful discussions on the ECPL experimental technique and early data analysis, and Prof. Juan Pablo Correa-Baena for access to the Energy Materials Laboratory.

\section{Notes and references}

1.
H. J. Snaith, Nature Materials, 2018, 17, 372-376. J.-P. Correa-Baena, M. Saliba, T. Buonassisi, M. Grätzel, A. Abate, W. Tress and A. Hagfeldt, Science, 2017, 358, 739. J. Y. Kim, J.-W. Lee, H. S. Jung, H. Shin and N.-G. Park, Chemical Reviews, 2020, 120, 7867-7918.

M. M. Lee, J. Teuscher, T. Miyasaka, T. N. Murakami and H. J. Snaith, Science, 2012, 338, 643.

Z.-K. Tan, R. S. Moghaddam, M. L. Lai, P. Docampo, R. Higler, F. Deschler, M. Price, A. Sadhanala, L. M. Pazos, D. Credgington, F. Hanusch, T. Bein, H. J. Snaith and R. H. Friend, Nature Nanotechnology, 2014, 9, 687-692.

K. Ji, M. Anaya, A. Abfalterer and S. D. Stranks, Advanced Optical Materials, 2021, n/a, 2002128.

G. Xing, N. Mathews, S. S. Lim, N. Yantara, X. Liu, D. Sabba, M. Grätzel, S. Mhaisalkar and T. C. Sum, Nature Materials, 2014, 13, 476-480.

B. R. Sutherland and E. H. Sargent, Nature Photonics, 2016, 10, 295-302.

https://www.nrel.gov/pv/cell-efficiency.html, (accessed March 30, 2021).

Y. Rakita, I. Lubomirsky and D. Cahen, Materials Horizons, 2019, 6, 1297-1305.

D. Shi, V. Adinolfi, R. Comin, M. Yuan, E. Alarousu, A. Buin, Y. Chen, S. Hoogland, A. Rothenberger, K. Katsiev, Y. Losovyj, X. Zhang, P. A. Dowben, O. F. Mohammed, E. H. Sargent and O. M. Bakr, Science, 2015, 347, 519.

M. Stolterfoht, C. M. Wolff, J. A. Márquez, S. Zhang, C. J. Hages, D. Rothhardt, S. Albrecht, P. L. Burn, P. Meredith, T. Unold and D. Neher, Nature Energy, 2018, 3, 847-854.

P. Schulz, D. Cahen and A. Kahn, Chemical Reviews, 2019, 119, 3349-3417.

A.-F. Castro-Méndez, J. Hidalgo and J.-P. Correa-Baena, Advanced Energy Materials, 2019, 9, 1901489.

S. G. Motti, M. Gandini, A. J. Barker, J. M. Ball, A. R. Srimath Kandada and A. Petrozza, ACS Energy Letters, 2016, 1, 726730.

J. S. W. Godding, A. J. Ramadan, Y.-H. Lin, K. Schutt, H. J. Snaith and B. Wenger, Joule, 2019, 3, 2716-2731.

D. W. deQuilettes, S. Koch, S. Burke, R. K. Paranji, A. J. Shropshire, M. E. Ziffer and D. S. Ginger, ACS Energy Letters, 2016, 1, 438-444.

W. Shockley and W. T. Read, Physical Review, 1952, 87, 835-842.

R. N. Hall, Physical Review, 1952, 87, 387-387.

S. D. Stranks, V. M. Burlakov, T. Leijtens, J. M. Ball, A. Goriely and H. J. Snaith, Physical Review Applied, 2014, 2, 034007.

A. Dobrovolsky, A. Merdasa, J. Li, K. Hirselandt, E. L. Unger and I. G. Scheblykin, The Journal of Physical Chemistry Letters, 2020, 11, 1714-1720.

V. S. Chirvony, S. González-Carrero, I. Suárez, R. E. Galian, M. Sessolo, H. J. Bolink, J. P. Martínez-Pastor and J. Pérez- 
Prieto, The Journal of Physical Chemistry C, 2017, 121, 45. 13381-13390.

23. V. S. Chirvony and J. P. Martínez-Pastor, The Journal of Physical Chemistry Letters, 2018, 9, 4955-4962.

24. D. W. deQuilettes, K. Frohna, D. Emin, T. Kirchartz, V. Bulovic, D. S. Ginger and S. D. Stranks, Chemical Reviews, 2019, 119, 11007-11019.

25. S. Tan, I. Yavuz, M. H. Weber, T. Huang, C.-H. Chen, R. Wang, H.-C. Wang, J. H. Ko, S. Nuryyeva, J. Xue, Y. Zhao, K.H. Wei, J.-W. Lee and Y. Yang, Joule, 2020, 4, 2426-2442. A. R. Srimath Kandada, S. Neutzner, V. D'Innocenzo, F. Tassone, M. Gandini, Q. A. Akkerman, M. Prato, L. Manna, A. Petrozza and G. Lanzani, Journal of the American Chemical Society, 2016, 138, 13604-13611.

27. D. Rosen, A. G. Doukas, Y. Budansky, A. Katz and R. R. Alfano, Applied Physics Letters, 1981, 39, 935-937.

28. D. von der Linde, J. Kuhl and E. Rosengart, Journal of Luminescence, 1981, 24-25, 675-678.

29. M. B. Johnson, T. C. McGill and A. T. Hunter, Journal of Applied Physics, 1988, 63, 2077-2082.

30. M. B. Johnson, T. C. McGill and N. G. Paulter, Applied Physics Letters, 1989, 54, 2424-2426.

31. M. Borgwardt, P. Sippel, R. Eichberger, M. P. Semtsiv, W. T. Masselink and K. Schwarzburg, Journal of Applied Physics, 2015, 117, 215702.

S. Pau, J. Kuhl, M. A. Khan and C. J. Sun, Physical Review B 1998, 58, 12916-12919.

33. M. Jo/rgensen and J. M. Hvam, Applied Physics Letters, 1983, 43, 460-462.

34. H. Hirori, K. Matsuda, Y. Miyauchi, S. Maruyama and Y. Kanemitsu, Physical Review Letters, 2006, 97, 257401.

Y. Miyauchi, K. Matsuda and Y. Kanemitsu, Physical Review B, 2009, 80, 235433.

36. S. Jariwala, S. Burke, S. Dunfield, C. Shallcross, M. Taddei, J. Wang, G. Eperon, N. Armstrong, J. Berry and D. S. Ginger, arXiv:2006.04025, 2020

37. K. Schötz, A. M. Askar, W. Peng, D. Seeberger, T. P. Gujar M. Thelakkat, A. Köhler, S. Huettner, O. M. Bakr, K. Shankar and F. Panzer, Journal of Materials Chemistry C, 2020, 8 2289-2300.

P. Fassl, V. Lami, F. J. Berger, L. M. Falk, J. Zaumseil, B. S Richards, I. A. Howard, Y. Vaynzof and U. W. Paetzold, Matter, 2021,

DOI: https://doi.org/10.1016/j.matt.2021.01.019.

39. D. W. de Quilettes, S. M. Vorpahl, S. D. Stranks, H. Nagaoka, G. E. Eperon, M. E. Ziffer, H. J. Snaith and D. S. Ginger, Science, 2015, 348, 683.

40. A. L. Alvarado-Leaños, D. Cortecchia, G. Folpini, A. R. Srimath Kandada and A. Petrozza, Advanced Optical Materials, 2021, n/a, 2001773.

41. R. L. Milot, G. E. Eperon, H. J. Snaith, M. B. Johnston and L. M. Herz, Advanced Functional Materials, 2015, 25, 62186227.

42. F. Ruf, M. F. Aygüler, N. Giesbrecht, B. Rendenbach, A Magin, P. Docampo, H. Kalt and M. Hetterich, APL Materials, 2019, 7, 031113.

43. S. Sun, T. Salim, N. Mathews, M. Duchamp, C. Boothroyd, G. Xing, T. C. Sum and Y. M. Lam, Energy \& Environmental Science, 2014, 7, 399-407.

44. Y. Yamada, T. Nakamura, M. Endo, A. Wakamiya and Y. Kanemitsu, IEEE Journal of Photovoltaics, 2015, 5, 401-405.
T. J. Savenije, C. S. Ponseca, L. Kunneman, M. Abdellah, K. Zheng, Y. Tian, Q. Zhu, S. E. Canton, I. G. Scheblykin, T. Pullerits, A. Yartsev and V. Sundström, The Journal of Physical Chemistry Letters, 2014, 5, 2189-2194.

K. Wu, A. Bera, C. Ma, Y. Du, Y. Yang, L. Li and T. Wu, Physical Chemistry Chemical Physics, 2014, 16, 2247622481

D. A. Valverde-Chávez, C. S. Ponseca, C. C. Stoumpos, A. Yartsev, M. G. Kanatzidis, V. Sundström and D. G. Cooke, Energy \& Environmental Science, 2015, 8, 3700-3707.

M. E. Ziffer, J. C. Mohammed and D. S. Ginger, ACS Photonics, 2016, 3, 1060-1068.

49. S. Jariwala, H. Sun, G. W. P. Adhyaksa, A. Lof, L. A. Muscarella, B. Ehrler, E. C. Garnett and D. S. Ginger, Joule, 2019, 3, 3048-3060.

50. D. W. deQuilettes, S. Jariwala, S. Burke, M. E. Ziffer, J. T. W. Wang, H. J. Snaith and D. S. Ginger, ACS Nano, 2017, 11, 11488-11496.

51. T. R. Hopper, A. Gorodetsky, J. M. Frost, C. Müller, R Lovrincic and A. A. Bakulin, ACS Energy Letters, 2018, 3, 2199-2205.

52. Y.-H. Hsieh, B.-W. Hsu, K.-N. Peng, K.-W. Lee, C. W. Chu, S.W. Chang, H.-W. Lin, T.-J. Yen and Y.-J. Lu, ACS Nano, 2020, 14, 11670-11676.

53. K. Kobbekaduwa, S. Shrestha, P. Adhikari, E. Liu, L. Coleman, J. Zhang, Y. Shi, Y. Zhou, Y. Bekenstein, F. Yan, A. M. Rao, H. Tsai, M. C. Beard, W. Nie and J. Gao, Nature Communications, 2021, 12, 1636 . 